\title{
Effective Geometry
}

\author{
M. Novello and S. E. Perez Bergliaffa \\ Centro Brasileiro de Pesquisas Físicas, \\ Rua Dr. Xavier Sigaud 150, \\ Urca 22290-180 Rio de Janeiro, RJ - Brazil
}

\begin{abstract}
We introduce the concept of effective geometry by studying several systems in which it arises naturally. As an example of the power and conciseness of the method, it is shown that a flowing dielectric medium with a linear response to an external electric field can be used to generate an analog geometry that has many of the formal properties of a Schwarzschild black hole for light rays, in spite of birefringence. The surface gravity of this analog black hole has a contribution that depends only on the dielectric properties of the fluid (in addition to the usual term dependent on the acceleration). This term may be give a hint to a new mechanism to increase the temperature of Hawking radiation.
\end{abstract}

\section{Contribution to the Proceedings of the $X^{\text {th }}$ Brazilian School of Gravitation and Cosmology, to be published by AIP.}

\section{INTRODUCTION}

In recent years, there has been a lot of interest in models that mimic in the laboratory some features of gravitation 1]. These models are built using systems that sometimes look (deceivingly) simple, and are very different in nature: ordinary nonviscous fluids, superfluids, flowing and non-flowing dielectrics, non-linear electromagnetism in vacuum, and Bose-Einstein condensates (see 2] for a complete list of references). The underlying physics in all these cases is the same: the behaviour of the fluctuations around a background solution is governed by an "effective metric". More precisely, the particles associated to the perturbations do not follow geodesics of the background spacetime but of a Lorentzian geometry described by the effective metric, which depends on the background solution. This allows a rather complete analogy of some kinematical aspects of general relativity [3], but not of its dynamical features (see however [2, 4]).

Using this analogy, the geometrical tools of General Relativity can be used to study some condensed matter systems 5]. More important perhaps is the fact that the analogy has permitted the simulation of several configurations of the gravitational field, such as wormholes and closed space-like curves for photons in nonlinear electrodynamics [7, 8], and warped spacetimes for phonons [9]. Particular attention has been paid to yet another configuration, namely analog black holes, because these would emit Hawking radiation exactly as gravitational black holes do, and they are obviously much easier to generate in the laboratory than their astrophysical counterpart. The fact that analog black holes emit thermal radiation was shown first by Unruh in the case of dumb black holes 10, and it is the prospect of observing this radiation (thus testing the hypothesis that the thermal emission is independent of the physics at arbitrarily short wavelengths [10]) that motivates the quest for a realization of analog black holes in the laboratory. Let us emphasize that the actual observation of the radiation is a difficult task from the point of view of the experiment, if only because of the extremely low temperatures involved. In the case of a quasi one-dimensional flow of a Bose-Einstein condensate for instance, the temperature of the radiation would be around $70 \mathrm{nK}$, which is comparable but lower than the temperature needed form the condensate [11].

We shall begin by presenting in Sect $\Pi$ the basics of the idea of the effective geometry by giving a sketch of an example given many years ago by W. Gordon 12. Then we shall move on to the more interesting case of nonlinear electromagnetism, where we introduce the mathematical tool of surface discontinuity. In Sect. III] we shall analyze another example: photons in a flowing dielectric medium. We shall see that, in analogy to the most general nonlinear 
electromagnetic case, the photons experience bi-refringence and bi-metricity. Then we demonstrate in Sect. IV that it is possible to build a static and spherically symmetric analog black hole, generated by a flowing isotropic dielectric that depends on an applied electric field. We give a specific example in Sect $\bar{V}$ in which the radius of the horizon and the temperature depend on three parameters (the zeroth order permittivity, the charge that generates the external field, and the linear susceptibility) instead of depending only on the zeroth order permittivity. As we shall show in Sect VI another feature of this black hole is that there is a new term in the surface gravity (and hence in the temperature of Hawking radiation), in addition to the usual term proportional to the acceleration of the fluid. This new term depends exclusively on the dielectric properties of the fluid, and it might give an opportunity to get Hawking radiation with temperature higher than that reported up to date.

\section{THE EFFECTIVE METRIC}

Historically, the first example of the idea of effective metric was presented by W. Gordon in 1923 [12]. In modern language, the wave equation for the propagation of light in a moving nondispersive medium, with slowly varying refractive index $n$ and 4 -velocity $u^{\mu}$ is given by

$$
\left[\partial_{\alpha} \partial^{\alpha}+\left(n^{2}-1\right)\left(u^{\alpha} \partial_{\alpha}\right)^{2}\right] F_{\mu \nu}=0
$$

Note that in this equation the components of $F_{\mu \nu}$ are not coupled. Consequently, the propagation will be the same independently of the polarization. In other words, there is no bi-refringence in moving media with constant refraction index (we shall see later that this is not the case if $n$ is a function of the coordinates). Taking the geometrical optics limit, with the eikonal Ansatz, given by $F_{\mu \nu}=\mathcal{F}_{\mu \nu} e^{i(\vec{k} \cdot \vec{x}-\omega t)}$, the Hamilton-Jacobi equation for light rays can be written as $g^{\mu \nu} k_{\mu} k_{\nu}=0$ (see [13] for details), where

$$
g^{\mu \nu}=\eta^{\mu \nu}+\left(n^{2}-1\right) u^{\mu} u^{\nu}
$$

is the effective metric for this problem. It must be remarked that only photons in the geometric optics approximation move on geodesics of $g^{\mu \nu}$ : the particles that compose the fluid couple instead to the background Minkowskian metric (in fact, the dynamics of the fluid is described by Euler's equation, and hence the background spacetime seen by the fluid particles is Newtonian).

Let us study now in detail the example of nonlinear electromagnetism. We start with the action

$$
S=\int \sqrt{-\gamma} L(F) d^{4} x
$$

where the invariant $F$ is given by $F \equiv F^{\mu \nu} F_{\mu \nu}$ [30], and $L$ is an arbitrary function of $F$. Notice that $\gamma$ is the determinant of the background metric, which we take in the following to be that of flat spacetime. However, the same techniques can be applied when the background is curved (see for instance [14]). Varying this action w.r.t. the potential $A_{\mu}$, related to the field by the expression $F_{\mu \nu}=A_{\mu, \nu}-A_{\nu, \mu}$, we obtain the Euler-Lagrange equations of motion (EOM):

$$
\left(\sqrt{-\gamma} L_{F} F^{\mu \nu}\right)_{; \nu}=0
$$

where $L_{F}$ is the functional derivative $L_{F} \equiv \frac{\delta L}{\delta F}$. In the particular case of a linear dependence of the Lagrangian with the invariant $F$ we recover Maxwell's EOM.

As mentioned in the Introduction, we would like to study the behaviour of perturbations of these EOM around a fixed background solution. In particular, we shall be interested in the causal structure inherent to the EOM (3). This structure is described by the characteristics of the EOM [15]. Instead of using the infinite-momentum limit of the eikonal approximation [17], we shall use a more elegant method set out by Hadamard [18]. In this method, the propagation of low-energy photons is studied by following the evolution of the wave front (i.e. the characteristic surface), through which the electromagnetic field is continuous but its first derivative is not. To be specific, let $\Sigma$ be the surface of discontinuity defined by the equation

$$
\Sigma\left(x^{\mu}\right)=\text { constant. }
$$

The discontinuity of a function $J$ through $\Sigma$ will be represented by $[J]_{\Sigma}$, and its definition is

$$
[J]_{\Sigma} \equiv \lim _{\delta \rightarrow 0^{+}}\left(\left.J\right|_{\Sigma+\delta}-\left.J\right|_{\Sigma-\delta}\right)
$$


The discontinuities of the field and its first derivative are given by

$$
\left[F_{\mu \nu}\right]_{\Sigma}=0, \quad\left[F_{\mu \nu, \lambda}\right]_{\Sigma}=f_{\mu \nu} k_{\lambda}
$$

where the vector $k_{\lambda}$ is nothing but the normal to the surface $\Sigma$, that is, $k_{\lambda}=\Sigma_{, \lambda}$.

To set the stage for the nonlinear case, let us first discuss the causal properties of Maxwell's electrodynamics, for which $L_{F}=$ const. The EOM then reduces to $F_{, \nu}^{\mu \nu}=0$, and taking its discontinuity we get

$$
f^{\mu \nu} k_{\nu}=0
$$

The other Maxwell equation is given by $F_{\mu \nu}^{*}, \nu=0$ or equivalently,

$$
F_{\mu \nu, \lambda}+F_{\nu \lambda, \mu}+F_{\lambda \mu, \nu}=0
$$

The discontinuity of this equation yields

$$
f_{\mu \nu} k_{\lambda}+f_{\nu \lambda} k_{\mu}+f_{\lambda \mu} k_{\nu}=0
$$

Multiplying this equation by $k^{\lambda}$ gives

$$
f_{\mu \nu} k^{2}+f_{\nu \lambda} k^{\lambda} k_{\mu}+f_{\lambda \mu} k^{\lambda} k_{\nu}=0
$$

where $k^{2} \equiv k_{\mu} k_{\nu} \gamma^{\mu \nu}$. Using the orthogonality condition from Eqn.(5) it follows that

$$
f^{\mu \nu} k^{2}=0
$$

Since the tensor associated to the discontinuity cannot vanish (we are assuming that there is a true discontinuity!) we conclude that the surface of discontinuity is null w.r.t. the metric $\gamma^{\mu \nu}$. That is,

$$
k_{\mu} k_{\nu} \gamma^{\mu \nu}=0
$$

(compare with Eqn.(10). It follows that $k_{\lambda, \mu} k^{\lambda}=0$, and since the vector of discontinuity is a gradient,

$$
k_{\mu, \lambda} k^{\lambda}=0
$$

This shows that the propagation of discontinuities of the electromagnetic field, in the case of Maxwell's equations (which are linear), is along the null geodesics of the Minkowski background metric.

Let us apply the same technique to the case of a nonlinear Lagrangian for the electromagnetic field, given by $L(F)$. Taking the discontinuity of the EOM (3), we get

$$
L_{F} f^{\mu \nu} k_{\nu}+2 a L_{F F} F^{\mu \nu} k_{\nu}=0,
$$

where we defined the quantity $a$ by $F^{\alpha \beta} f_{\alpha \beta} \equiv a$. Note that contrary to the linear case in which the discontinuity tensor $f_{\mu \nu}$ is orthogonal to the propagation vector $k^{\mu}$, here there is a complicated relation between the vector $f^{\mu \nu} k_{\nu}$ and quantities dependent on the background field. This is the origin of a more involved expression for the evolution of the discontinuity vector, as we shall see next. Multiplying equation (8) by $F^{\mu \nu}$ we obtain

$$
a k^{2}+F^{\mu \nu} f_{\nu \lambda} k^{\lambda} k_{\mu}+F^{\mu \nu} f_{\lambda \mu} k^{\lambda} k_{\nu}=0
$$

Now we substitute in this equation the term $f^{\mu \nu} k_{\nu}$ from Eqn.(12), and we arrive at the expression

$$
a k^{2}-2 \frac{L_{F F}}{L_{F}} a\left(F^{\mu \lambda} k_{\mu} k_{\lambda}-F^{\lambda \mu} k_{\mu} k_{\lambda}\right),
$$

which can be written as $g^{\mu \nu} k_{\mu} k_{\nu}=0$, where

$$
g^{\mu \nu}=\gamma^{\mu \nu}-4 \frac{L_{F F}}{L_{F}} F^{\mu \nu}
$$

We then conclude that 
The low-energy photons of a nonlinear theory of electrodynamics with $L=$ $L(F)$ do not propagate on the null cones of the background metric but on the null cones of an effective metric, generated by the self-interaction of the electromagnetic field.

This statement is always true in case of Lagrangians depending only of the invariant $F$. For Lagrangians that depend also of $F^{*}$, there may be some special cases in which the propagation coincides with that in Minkowski $[\underline{6}$. Another feature of the more general case $L=L\left(F, F^{*}\right)$ is that bi-refringence is present. That is, each of the two polarization states of the photon has its own dispersion relation. In some special cases, there is also bi-metricity (one effective metric for each polarization state). Some more special cases (such as Born-Infeld electrodynamics) even exhibit only a single metric [16, 17]. Several of these features are present in our next example.

\section{EFFECTIVE METRIC(S) IN THE PRESENCE OF A DIELECTRIC}

Let us now move to another interesting case where the effective geometry is useful to study the causal properties of low-energy photons. We shall analyze the propagation of such photons in a nonlinear medium (see Ref. [23] for details and notation). Let us define first the antisymmetric tensors $F_{\mu \nu}$ and $P_{\mu \nu}$, which are convenient to represent the electromagnetic field when material media are present. These tensors can be expressed in terms of the strengths $(E, H)$ and the excitations $(D, B)$ of the electric and magnetic fields as

$$
\begin{aligned}
& F_{\mu \nu}=v_{\mu} E_{\nu}-v_{\nu} E_{\mu}-\eta_{\mu \nu}{ }^{\alpha \beta} v_{\alpha} B_{\beta}, \\
& P_{\mu \nu}=v_{\mu} D_{\nu}-v_{\nu} D_{\mu}-\eta_{\mu \nu}{ }^{\alpha \beta} v_{\alpha} H_{\beta} .
\end{aligned}
$$

where $v_{\mu}$ represents the 4 -velocity of an arbitrary observer (which we will take later as comoving with the fluid). The Levi-Civita tensor introduced above is defined in such way that $\eta^{0123}=+1$ in Cartesian coordinates. Since the electric and magnetic fields are spacelike vectors, we shall use the notation $E^{\alpha} E_{\alpha} \equiv-E^{2}, H^{\alpha} H_{\alpha} \equiv-H^{2}$. We will consider here media with properties determined only by the tensors $\epsilon_{\alpha \beta}$ and $\mu_{\alpha \beta}$ (i.e. media with null magneto-electric tensor), which relate the electromagnetic excitations to the field strengths by the constitutive laws,

$$
D_{\alpha}=\epsilon_{\alpha}{ }^{\beta}(E, H) E_{\beta}, \quad B_{\alpha}=\mu_{\alpha}{ }^{\beta}(E, H) H_{\beta} .
$$

In order to get the effective metric, we shall use Hadamard's method [18] as in the previous section. By taking the discontinuity of the field equations ${ }^{*} F^{\mu \nu}{ }_{, \nu}=0$ and $P^{\mu \nu}{ }_{, \nu}=0$, and assuming that

$$
\epsilon^{\mu \beta}=\epsilon(E)\left(\gamma^{\mu \beta}-v^{\mu} v^{\beta}\right),
$$

and

$$
\mu^{\mu \beta}=\mu_{0}\left(\gamma^{\mu \beta}-v^{\mu} v^{\beta}\right),
$$

with $\mu_{0}=$ const., we get the following equations:

$$
\begin{gathered}
\epsilon(k . e)-\frac{\epsilon^{\prime}}{E}(E . e)(k . E)=0, \\
\mu_{0}(k . h)=0, \\
\epsilon(k . v) e^{\mu}-\frac{\epsilon^{\prime}}{E} E^{\alpha} e_{\alpha}(k . v) E^{\mu}+\eta^{\mu \nu \alpha \beta} k_{\nu} v_{\alpha} h_{\beta}=0, \\
\mu_{0}(k \cdot v) h^{\mu}-\eta^{\mu \nu \alpha \beta} k_{\nu} v_{\alpha} e_{\beta}=0,
\end{gathered}
$$

where $k^{\mu}$ is the wave propagation vector, $\epsilon^{\prime}$ is the derivative of $\epsilon$ w.r.t. $E$, and

$$
\left[E_{\mu, \lambda}\right]_{\Sigma}=e_{\mu} k_{\lambda}, \quad\left[H_{\mu, \lambda}\right]_{\Sigma}=h_{\mu} k_{\lambda} .
$$


Note in particular that Eqn.(19) shows that the vectors $k^{\mu}$ and $e^{\mu}$ are not always orthogonal, as would be the case if $\epsilon^{\prime}$ was zero. Substituting Eqn.(22) in (21), we get

$$
Z^{\mu \beta} e_{\beta}=0
$$

where the matrix $Z$ is given by

$$
Z^{\mu \beta}=\left[k^{2}+(k . v)^{2}\left(\mu_{0} \epsilon-1\right)\right] \gamma^{\mu \beta}-\mu_{0} \frac{\epsilon^{\prime}}{E}(k . v)^{2} E^{\mu} E^{\beta}+(v . k)\left(v^{\mu} k^{\beta}+k^{\mu} v^{\beta}\right)-\left[\epsilon \mu_{0}(k . v)+k^{2}\right] v^{\mu} v^{\beta}-k^{\mu} k^{\beta} .
$$

Non-trivial solutions of Eqn.(23) can be found only for cases in which $\operatorname{det}\left|Z^{\mu \beta}\right|=0$ ( this condition is a generalization of the well-known Fresnel equation [19]).

Eqn.(23) can be solved by expanding $e_{\nu}$ as a linear combination of the four linearly independent vectors $v_{\nu}, E_{\nu}, k_{\nu}$ and $\eta_{\alpha \beta \mu \nu} v^{\alpha} E^{\beta} k^{\mu}[31]$. That is,

$$
e_{\nu}=\alpha E_{\nu}+\beta \eta_{\alpha \lambda \mu \nu} v^{\alpha} E^{\lambda} k^{\mu}+\gamma k_{\nu}+\delta v_{\nu}
$$

Notice that taking the discontinuity of $E_{, \lambda}^{\mu}$ we can show that $(e . v)=0$. This restriction imposes a relation between the coefficients of Eqn.(25):

$$
\delta=-\gamma(k \cdot v)
$$

With the expression given in Eqn.(25), Eqn.(23) reads

$$
\begin{aligned}
\alpha\left[k^{2}-\left(1-\mu_{0}(\epsilon E)^{\prime}\right)(k . v)^{2}\right]-\gamma\left[\mu_{0}(k . v)^{2} \frac{1}{E} \epsilon^{\prime} E^{\alpha} k_{\alpha}\right]=0 & \\
\alpha E^{\mu} k_{\mu}+\gamma\left(1-\mu_{0} \epsilon\right)(k . v)^{2}+\delta(k . v) & =0 \\
\alpha(k . v) E^{\mu} k_{\mu}+\gamma(k . v) k^{2}+\delta\left[k^{2}+\mu_{0} \epsilon(k . v)^{2}\right] & =0 \\
\beta\left[k^{2}-\left(1-\mu_{0} \epsilon\right)(k . v)^{2}\right] & =0
\end{aligned}
$$

The solution of this system results in the following dispersion relations:

$$
\begin{aligned}
& k_{-}^{2}=(k . v)^{2}\left[1-\mu_{0}(\epsilon E)^{\prime}\right]+\frac{1}{\epsilon E} \epsilon^{\prime} E^{\alpha} E^{\beta} k_{\alpha} k_{\beta}, \\
& k_{+}^{2}=\left[1-\mu_{0} \epsilon(E)\right](k . v)^{2} .
\end{aligned}
$$

They correspond to the propagation modes

$$
\begin{aligned}
e_{\nu}^{-} & =\rho^{-}\left\{\mu_{0} \epsilon(k . v)^{2} E_{\nu}+E^{\alpha} k_{\alpha}\left[k_{\nu}-(k . v) v_{\nu}\right]\right\} \\
e_{\nu}^{+} & =\rho^{+} \eta_{\alpha \lambda \mu \nu} v^{\alpha} E^{\lambda} k^{\mu}
\end{aligned}
$$

where $\rho^{-}$and $\rho^{+}$are arbitrary constants. The labels "+" and "-" refer to the ordinary and extraordinary rays, respectively. Eqns. (26) and (27) govern the propagation of photons in the medium characterized by $\mu=\mu_{0}=$ const., and $\epsilon=\epsilon(E)$. They can be rewritten as $g_{ \pm}^{\mu \nu} k_{\mu} k_{\nu}=0$, where we have defined the effective geometries

$$
\begin{aligned}
& g_{(-)}^{\mu \nu}=\gamma^{\mu \nu}-\left[1-\mu_{0}(\epsilon E)^{\prime}\right] v^{\mu} v^{\nu}-\frac{1}{\epsilon E} \epsilon^{\prime} E^{\mu} E^{\nu} \\
& g_{(+)}^{\mu \nu}=\gamma^{\mu \nu}-\left[1-\mu_{0} \epsilon\right] v^{\mu} v^{\nu}
\end{aligned}
$$

The metric given by Eqn.(30) was derived first in 22], while the second metric very much resembles the metric obtained by Gordon [12] (see Eqn.(11). The difference is that in the case under consideration, $\epsilon$ is a function of the modulus of the external electric field, while Gordon worked with a constant permeability.

We see then that in this example each polarization state has its own dispersion relation (Eqns.(26) and (27)), so there is bi-refringence. There is also bi-metricity, because each type of photon moves according a different metric (see Eqns. (30) and (31). 


\section{THE ANALOG BLACK HOLE}

We shall show in this section that the system described by the effective metrics given by Eqns. (30)-(31) can be used to produce an analog black hole. It will be convenient to rewrite at this point the inverse of the effective metric given by Eqn.(30) using a different notation:

$$
g_{\mu \nu}^{(-)}=\gamma_{\mu \nu}-\frac{v_{\mu} v_{\nu}}{c^{2}}(1-f)+\frac{\xi}{1+\xi} l_{\mu} l_{\nu}
$$

where we have defined the quantities

$$
f \equiv \frac{1}{c^{2} \mu_{0} \epsilon(1+\xi)}, \quad \xi \equiv \frac{\epsilon^{\prime} E}{\epsilon}, \quad l_{\mu} \equiv \frac{E_{\mu}}{E} .
$$

Note that $\epsilon=\epsilon(E)$. We have introduced here the velocity of light $c$, which was set to 1 before. Taking a Minkowskian background in spherical coordinates, and

$$
v_{\mu}=\left(v_{0}, v_{1}, 0,0\right), \quad E_{\mu}=\left(E_{0}, E_{1}, 0,0\right),
$$

we get for the effective metric described by Eqn. (32),

$$
\begin{gathered}
g_{00}^{(-)}=1-\frac{v_{0}^{2}}{c^{2}}(1-f)+\frac{\xi}{1+\xi} l_{0}^{2}, \\
g_{11}^{(-)}=-1-\frac{v_{1}^{2}}{c^{2}}(1-f)+\frac{\xi}{1+\xi} l_{1}^{2}, \\
g_{01}^{(-)}=-\frac{v_{0} v_{1}}{c^{2}}(1-f)+\frac{\xi}{1+\xi} l_{0} l_{1},
\end{gathered}
$$

and $g_{22}^{(-)}$and $g_{33}^{(-)}$as in Minkowski spacetime. The vectors $v_{\mu}$ and $l_{\mu}$ satisfy the constraints

$$
\begin{gathered}
v_{0}^{2}-v_{1}^{2}=c^{2}, \\
l_{0}^{2}-l_{1}^{2}=-1, \\
v_{0} l_{0}-v_{1} l_{1}=0 .
\end{gathered}
$$

This system of equations can be solved in terms of $v_{1}$, and the result is

$$
\begin{gathered}
v_{0}^{2}=c^{2}+v_{1}^{2}, \\
l_{0}^{2}=\frac{v_{1}^{2}}{c^{2}}, \quad l_{1}^{2}=\frac{c^{2}+v_{1}^{2}}{c^{2}} .
\end{gathered}
$$

Now we can rewrite the metric in terms of $\beta \equiv v_{1} / c$, a definition which coincides with the usual one for small values of $v_{1}$. The explicit expression of the metric coefficients is:

$$
\begin{gathered}
g_{00}^{(-)}=\frac{1-\beta^{2}\left(c^{2} \mu_{0} \epsilon-1\right)}{c^{2} \mu_{0}\left(\epsilon+\epsilon^{\prime} E\right)}, \\
g_{01}^{(-)}=\beta \sqrt{1+\beta^{2}} \frac{1-c^{2} \mu_{0} \epsilon}{c^{2} \mu_{0}\left(\epsilon+\epsilon^{\prime} E\right)}, \\
g_{11}^{(-)}=\frac{\beta^{2}-c^{2} \mu_{0} \epsilon\left(1+\beta^{2}\right)}{c^{2} \mu_{0}\left(\epsilon+\epsilon^{\prime} E\right)} .
\end{gathered}
$$


From Eqn.(42) it is easily seen that, depending on the function $\epsilon(E)$, this metric has a horizon at $r=r_{h}$, given by the condition $g_{00}\left(r_{h}\right)=0$ or equivalently,

$$
\left.\left(c^{2} \mu_{0} \epsilon-\frac{1}{\beta^{2}}\right)\right|_{r_{h}}=1
$$

The metric given above resembles the form of Schwarzschild's solution in Painlevé-Gullstrand coordinates [24, 25]:

$$
d s^{2}=\left(1-\frac{2 G M}{r}\right) d t^{2} \pm 2 \sqrt{\frac{2 G M}{r}} d r d t-d r^{2}-r^{2} d \Omega^{2}
$$

With the coordinate transformation

$$
d t_{P}=d t_{S} \mp \frac{\sqrt{2 G M / r}}{1-\frac{2 G M}{r}} d r
$$

the line element given in Eqn.(46) can be written in Schwarzschild's coordinates. The "+" sign covers the future horizon and the black hole singularity.

The effective metric given by Eqns.(42)-(44) looks like the metric in Eqn.(46) 32]. In fact, it can be written in Schwarzschild's coordinates, with the coordinate change

$$
d t_{P G}=d t_{S}-\frac{g_{01}(r)}{g_{00}(r)} d r
$$

Using this transformation with the metric coefficients given in Eqns.42) and (43), we get the expression of $g_{11}^{(-)}$in Schwarzschild coordinates:

$$
g_{11}^{(-)}=-\frac{\epsilon(E)}{\left(1-\beta^{2}\left[c^{2} \mu_{0} \epsilon(E)-1\right]\right)\left(\epsilon(E)+\epsilon(E)^{\prime} E\right)} .
$$

Note that $g_{01}^{(-)}$is zero in the new coordinate system, while $g_{00}^{(-)}$is still given by Eqn. (42). Consequently, the position of the horizon does not change, and is still given by Eqn.(45).

Working in Painlevé-Gullstrand coordinates, we have shown that the metric for the "_" polarization describes a Schwarzschild black hole if Eqn.(45) has a solution. Afterwards we have rewritten the "_" metric in more familiar coordinates. By means of similar calculations, it can be shown that photons with the other polarization "see" the metric (in Schwarzschild coordinates) given by

$$
\begin{gathered}
g_{00}^{(+)}=\frac{1+\beta^{2}\left(1-c^{2} \mu_{0} \epsilon(E)\right)}{c^{2} \mu_{0} \epsilon(E)}, \\
g_{11}^{(+)}=-\frac{1}{1+\beta^{2}\left(1-c^{2} \mu_{0} \epsilon(E)\right)} .
\end{gathered}
$$

It is important to stress then that the horizon is located at $r_{h}$ given by Eqn. (45) for photons with any polarization. Moreover, the motion of the photons in both geometries will be qualitatively the same, as we shall show below.

\section{AN EXAMPLE}

We have not specified up to now the functions $\epsilon(E)$ and $E(r)$ that determine the dependence of the coefficients of the effective metrics with the coordinate $r$. From now on we assume a linear $\epsilon(E)$, a type of behaviour which is exhibited for instance by electrorheological fluids [29]. Specifically, we take

$$
\epsilon(E)=\epsilon_{0}\left(\bar{\chi}+\chi^{(2)} E(r)\right)
$$

with $\bar{\chi}=1+\chi^{(1)}$. The nontrivial Maxwell's equation then reads

$$
\left(\sqrt{-\gamma} \epsilon(r) F^{01}\right)_{, 1}=0
$$


Taking into account that $\left(F^{01}\right)^{2}=\frac{E^{2}}{c^{2}}$, we get

$$
F^{01}=\frac{-\bar{\chi} \pm \sqrt{\bar{\chi}^{2}+4 \chi^{(2)} Q / \epsilon_{0} r^{2}}}{2 c \chi^{(2)}} .
$$

as a solution of Eqn.(53) for a point source in a flat background in spherical coordinates. Let us consider a particular combination of parameters: $\chi^{(2)}>0, Q>0$ and the "+" sign in front of the square root in $F^{01}$, in such a way that $E>0$ for all $r$. To get more manageable expressions for the metric, it is convenient to define the function $\sigma(r)$ :

$$
E(r) \equiv \frac{\bar{\chi}}{2 \chi^{(2)}} \sigma(r)
$$

where

$$
\sigma(r)=-1+\frac{1}{r} \sqrt{r^{2}+q}
$$

and

$$
q=\frac{4 \chi^{(2)} Q}{\epsilon_{0} \bar{\chi}^{2}}
$$

In terms of $\sigma$, the metrics take the form

$$
\begin{gathered}
d s_{(-)}^{2}=\frac{2-\beta^{2}[\bar{\chi}(\sigma(r)+2)-2]}{2 \bar{\chi}(1+\sigma(r))} d \tau^{2}-\frac{2+\sigma(r)}{\left[2-\beta^{2}(\bar{\chi}(\sigma(r)+2)-2)\right](1+\sigma(r))} d r^{2}-r^{2} d \Omega^{2}, \\
d s_{(+)}^{2}=\frac{2-\beta^{2}[\bar{\chi}(\sigma(r)+2)-2]}{\bar{\chi}(2+\sigma(r))} d \tau^{2}-\frac{2}{2+\beta^{2}[2-\bar{\chi}(\sigma(r)+2)]} d r^{2}-r^{2} d \Omega^{2} .
\end{gathered}
$$

Notice that the $(t, r)$ sectors of these metrics are related by the following expression:

$$
d s_{(+)}^{2}=\Phi(r) d s_{(-)}^{2}
$$

where the conformal factor $\Phi$ is given by:

$$
\Phi=2 \frac{1+\sigma(r)}{2+\sigma(r)}
$$

We shall study next some features of the effective black hole metrics. It is important to remark that up to this point, the velocity of the fluid $v_{1}$ is completely arbitrary; it can even be a function of the coordinate $r$. We shall assume in the following that $v_{1}$ is a constant. This assumption, which will be lifted in Sect VI may seem rather restrictive but it helps to display the main features of the effective metrics in an easy way.

To study the motion of the photons in these geometries, we can use the technique of the effective potential. Standard manipulations (see for instance [26]) show that in the case of a static and spherically symmetric metric, the effective potential is given by

$$
V(r)=\varepsilon^{2}\left(1+\frac{1}{g_{00}(r) g_{11}(r)}\right)-\frac{L^{2}}{r^{2} g_{11}(r)}
$$

where $\varepsilon$ is the energy and $L$ the angular momentum of the photon.

In terms of $\sigma(r)$, and of the impact parameter $b^{2}=L^{2} / \varepsilon^{2}$, the "small" effective potential $v(r) \equiv V(r) / \varepsilon^{2}$ for the metric Eqn.(58) in Schwarzschild coordinates can be written as follows:

$$
v^{(-)}(r)=1-\frac{2(1+\sigma(r))^{2}}{2+\sigma(r)}-\frac{b^{2}}{r^{2}} \frac{\left(2-\beta^{2} \sigma(r)\right)(1+\sigma(r))}{2+\sigma(r)}
$$

A short calculation shows that $v^{(-)}$is a monotonically decreasing function of $\beta$. Consequently, we shall choose a convenient value of it, for the sake of illustrating the features of the effective potential. Figures (1) and (2) show the plots of the potential for the $(-)$ metric for several values of the relevant parameters. 


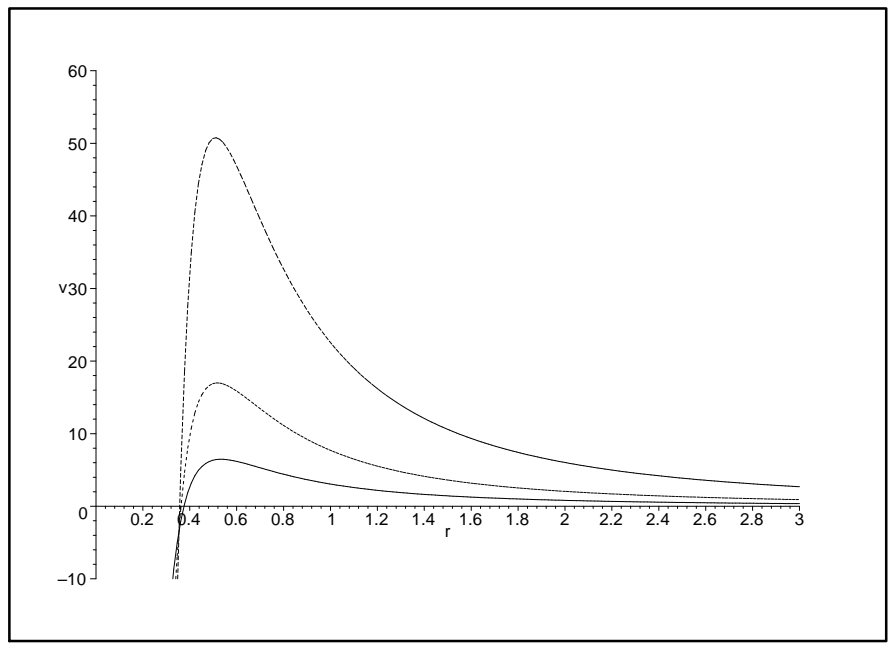

FIG. 1: Plot of the effective potential $v^{(-)}(r)$ for $q=1, b=1,3,5$ (starting from the lowest curve), and $\beta=0.5$.

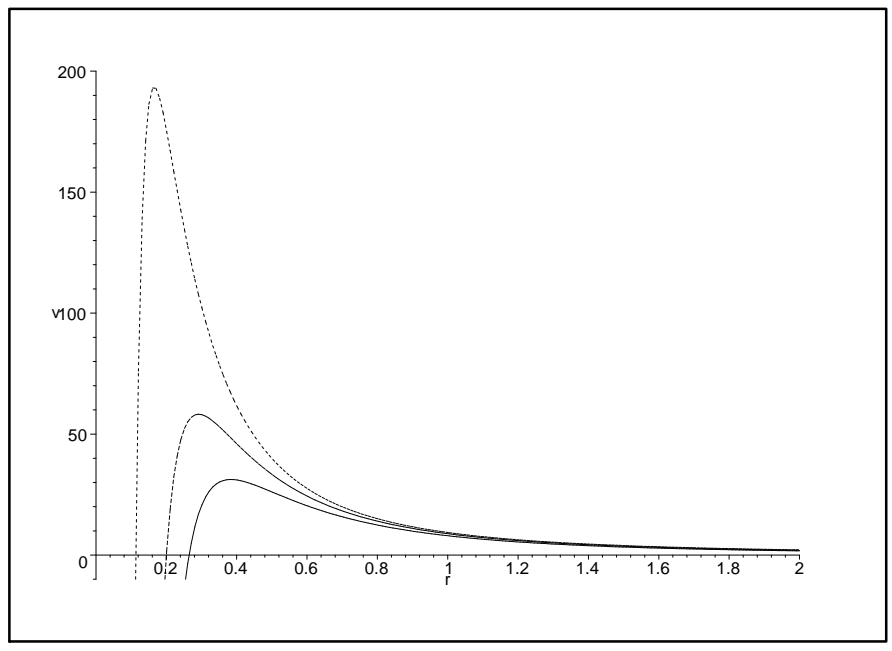

FIG. 2: Plot of the effective potential $v^{(-)}(r)$ for $b=3$, and $q=1,3,5$ (starting from the lowest curve), and $\beta=0.5$.

The effective potential for the Gordon-like metric can be obtained in the same way. From Eqns.(61) and (59) we get

$$
v^{(+)}(r)=1-\frac{2+\sigma(r)}{2}+\frac{b^{2}}{2 r^{2}}\left[2-\beta^{2} \sigma(r)\right] .
$$

The plots in Figures (3) and (4) show the dependence of $v^{(+)}(r)$ on the different parameters.

We see from these plots that, in the case of a constant flux velocity, the shape of the effective potential for both metrics qualitatively agrees with that for photons moving on the geometry of a Schwarzschild black hole (see for instance Ref.[26], pag. 143).

\section{SURFACE GRAVITY AND TEMPERATURE}

Let us now go back to the more general case of $\beta=\beta(r)$, and calculate the "surface gravity" of our analog black hole. We present first the results for the constant permittivity case. By setting $\epsilon^{\prime}(E) \equiv 0$ in the metrics Eqns. (30) and (31), we regain the example of constant index of refraction studied for instance in [21]. It is easy to show from 


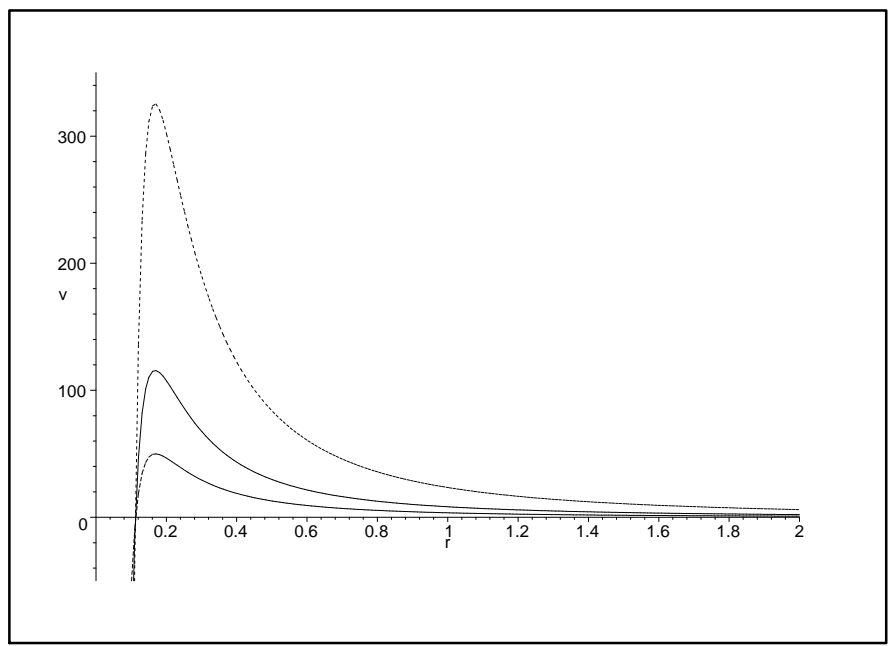

FIG. 3: Plot of the effective potential for the Gordon-like metric, for $q=1, b=1,3,5$ (starting from the lowest curve), and $\beta=0.5$.

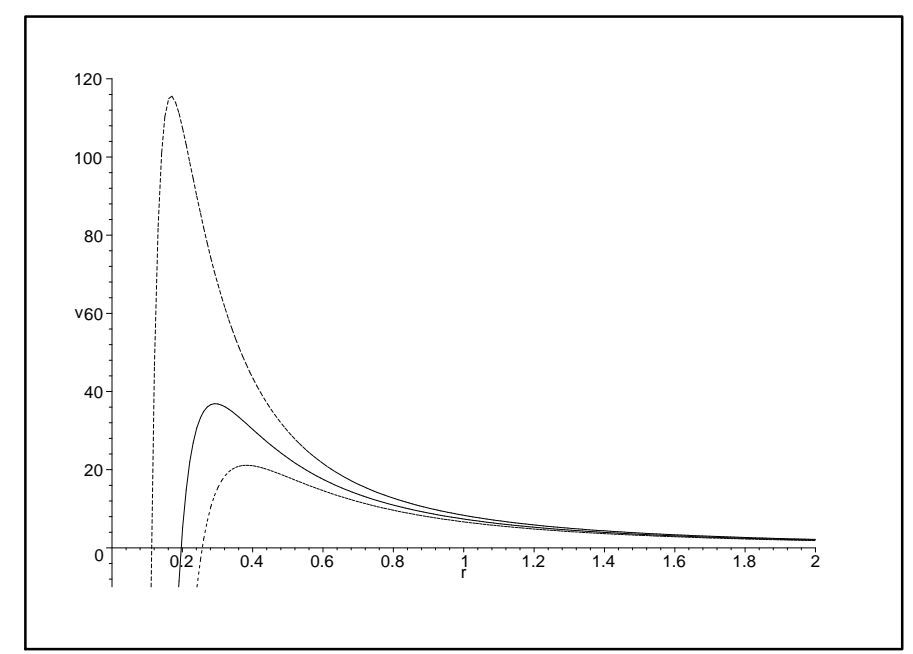

FIG. 4: Plot of the effective potential for the Gordon-like metric, for $b=3, q=1,3,5$.

Eqn.(45) that the horizon of the black hole in this case is given by

$$
\beta^{2}\left(r_{h}\right)=\frac{1}{\bar{\chi}-1}
$$

The "surface gravity" of a spherically symmetric analog black hole in Schwarszchild coordinates is given by [5]

$$
\kappa=\frac{c^{2}}{2} \lim _{r \rightarrow r_{h}} \frac{g_{00, r}}{\sqrt{\left|g_{11}\right| g_{00}}}
$$

For the metrics Eqns.(30) and (31) with $\epsilon=\epsilon_{0} \bar{\chi}$ and $r_{h}$ given by Eqn. (64), the analog surface gravity is

$$
\kappa=-\left.\frac{c^{2}}{2} \frac{1-\bar{\chi}}{\sqrt{\bar{\chi}}}\left(\beta^{2}\right)^{\prime}\right|_{r_{h}} .
$$

This equation can be rewritten in terms of the velocity of light in the medium and the refraction index, respectively given by

$$
c_{m}^{2}=\frac{1}{\mu_{0} \epsilon}, \quad n=\frac{c}{c_{m}} .
$$


The result is

$$
\kappa=\frac{c^{2}}{2} \frac{1-n^{2}}{n}\left(\beta^{2}\right)_{, r}
$$

In this expression we can see the influence of the dielectric properties of the fluid (through the index of refraction of the medium) and also of its dynamics through the physical acceleration in the radial direction, given by

$$
\left.a_{r}\right|_{r_{h}}=\left.\frac{c^{2}}{2}\left(\beta^{2}\right)^{\prime}\right|_{r_{h}},
$$

for $\beta^{2}\left(r_{h}\right) \ll 1$. This acceleration is a quantity that must be determined solving the equations of motion of the fluid. (Notice that if $\beta$ is set equal to 0 in Eqns.(42)-(44), we cease to have a black hole (this situation was analyzed in [16])).

Going back the the more general case of a linear permittivity, described by the metrics given by Eqns.(158) and (59), and considering that $\beta\left(r_{h}\right) \ll 1$, the radius of the horizon is [33]:

$$
r_{h}^{2}=\frac{q \bar{\chi}^{2}}{4} \beta^{4}\left(r_{h}\right) .
$$

Using the expressions given above, the result for the surface gravity of the "-" black hole for $\beta\left(r_{h}\right) \ll 1$ is

$$
\kappa^{(-)}=\left.\frac{c^{2}}{\beta}\left(\frac{1}{\bar{\chi} \sqrt{q}}-\frac{1}{2}\left(\beta^{2}\right)^{\prime}\right)\right|_{r_{h}} .
$$

This equation differs from the surface gravity of the case of constant permittivity (Eqn. (66) ) by the presence of a new term that does not depend on the acceleration of the fluid. To see where this new term comes from, we can go back to the definition of the surface gravity given in Eqn. 65 , and use the fact that in the high frequency limit the velocity of light and the index of refraction in a medium of variable $\epsilon$ are still given by Eqn. (67), replacing the constant permittivity by $\epsilon=\epsilon(E)$. The result is

$$
\kappa=\left.\left(\frac{c^{2}}{2} \frac{1-n^{2}(E)}{n(E)}\left(\beta^{2}\right)_{, r}+\frac{n(E) \epsilon(E)}{\epsilon(E)+\epsilon(E)^{\prime} E}\left(c_{m}^{2}\right)_{, r}\right)\right|_{r_{h}}
$$

In this expression, the first term is the generalization of the case $\epsilon=$ const. (compare with Eqn. 668), which mixes the acceleration of the fluid with its dielectric properties. On the other hand, the second term, which is the new term displayed in Eqn.(70), is related to the radial variation of the velocity of light in the medium. It is important to point out that the result exhibited in Eqn.(71) is parallel to that of dumb holes: Unruh found in that case [10] that the surface gravity for constant speed of sound is proportional to the acceleration of the fluid (as in the first term of Eqn.(171)). This was generalized by Visser [5], who showed that for a position-dependent velocity of sound a second term appears, coming from the gradients of the speed of sound, in analogy with the second term of Eqn. (711).

It is easy to show that the these results also apply to the black hole described by the Gordon-like metric. This is not surprising though, because of the conformal relation between the two metrics, given by Eqn.(60) [28].

Let us remark once more that the concept of temperature, and indeed that of effective geometry is valid in this context only for low-energy photons, i.e. photons with wavelengths long compared to the intermolecular spacing in the fluid. For shorter wavelengths, there would be corrections to the propagation dictated by the effective metric. However, results for other systems (such as dumb black holes [10] and Bose-Einstein condensates) suggest that the phenomenon of Hawking radiation is robust (i.e. independent of this "high-energy" physics). Consequently, it makes sense to talk about the temperature of the radiation in these systems.

At first sight it may seem that by choosing an appropriate material and a convenient value of the charge we could obtain a high value of the temperature of the radiation, given by

$$
T \equiv \frac{\hbar}{2 \pi k_{B} c} \kappa \approx 4 \times 10^{-21} \kappa \mathrm{Ks}^{2} / \mathrm{m} .
$$

However, the equation for the surface gravity can be rewritten as $\underline{34}$

$$
\kappa=\left.c^{2}\left(\frac{\beta}{2 r}-\beta_{, r}\right)\right|_{r_{h}} .
$$

We see then that, because $\beta\left(r_{h}\right) \ll 1$, the new term appearing in $\kappa$ is bound to be very small. In spite of this result, the emergence in the surface gravity of the term due to the variable velocity of light suggests that it may be worth to study if some media with nonlinear dependence on an external electromagnetic field can be used to generate analog black holes whose Hawking radiation could be measured in laboratory. 


\section{Acknowledgements}

The authors would like to thank CNPq and FAPERj for financial support.

[1] "Artificial Black Holes", Proceedings of the Workshop "Analog Models of General Relativity" (held at the Centro Brasileiro de Pesquisas Fisicas, Brazil, Oct. 2000), M. Novello, M. Visser, and G. Volovik (Eds), World Scientific (2002).

[2] A complete list of references can be found in C. Barcelo, S. Liberati, and M. Visser, Class. Quantum Grav.18, 3595 (2001).

[3] M. Visser, Phys. Rev. Lett. 80, 3439 (1998) .

[4] C. Barcelo, S. Liberati, M. Visser, Class. Quantum Grav.19, 2961 (2002).

[5] See M. Visser, Class. Quantum Grav. 15, 1767 (1998) and references therein.

[6] V. A. De Lorenci, Renato Klippert, M. Novello, J.M. Salim, Phys. Lett. B482, 134 (2000).

[7] F. Baldovin, M. Novello, S.E. Perez Bergliaffa, and J.M. Salim, Class. Quant. Grav. 17, 3265 (2000).

[8] M. Novello, J. M. Salim, V. A. De Lorenci, and E. Elbaz, Phys. Rev. D 63, 103516 (2001).

[9] "Warped spacetime for phonons moving in a perfect nonrelativistic fluid", U. Fischer and Matt Visser, gr-qc/0211029

[10] W. Unruh, Phys. Rev. Lett. 46, 1351 (1981), and Phys. Rev. D 51, 2827 (1995).

[11] Towards the observation of Hawking radiation in Bose-Einstein condensates, C. Barcelo, S. Liberati, and M. Visser, $\mathrm{gr}-\mathrm{qc} / 0110036$

[12] W. Gordon, Ann. Phys. (Leipzig), 72, 421 (1923).

[13] U. Leonhardt and P. Piwnicki, Phys. Rev. A 60, 4301 (1999).

[14] M. Novello, S.E. Perez Bergliaffa, J.M. Salim, Class. Quant. Grav. 17, 3821 (2000).

[15] Methods of Mathematical Physics, R. Courant and D. Hilbert, Wiley, John and Sons (1990).

[16] M. Novello, V. De Lorenci, J. Salim, and R. Klippert, Phys. Rev D61, 45001 (2000).

[17] Bi-refringence versus bi-metricity, M. Visser, C. Barcelo, and S. Liberati. Contribution to the Festschrift in honor of Mario Novello, to be published. gr-qc/0204017

[18] Leçons sur la propagation des ondes et les equations de l'hydrodynamique, J. Hadamard, Ed. Dunod, Paris, 1958.

[19] See for instance Electrodynamique des milieux continus, L. Landau and E. Lifshitz, Ed. Mir, Moscow (1969).

[20] C. Barcelo, S. Liberati, and M. Visser, Class. Quant. Grav. 18, 3595 (2001).

[21] U. Leonhardt and P. Piwnicki, Phys. Rev. Lett. 84, 822 (2000) and Phys. Rev. A 60, 4301 (1999). See the subsequent discussion in M. Visser, Phys. Rev. Lett. 85, 5252 (2000) and U. Leonhardt and P. Piwnicki, Phys. Rev. Lett. 85, 5253 (2000), R. Schutzhold, G. Plunien, and G. Soff, Phys. Rev. Lett. 88, 061101 (2002).

[22] M. Novello, J. Salim, Phys. Rev. D 63, 083511 (2001).

[23] Analog black holes in flowing dielectrics, M. Novello, S. Perez Bergliaffa, J. Salim, V. De Lorenci, and R. Klippert, gr-qc/0201061 To be published in Class. Quantum Grav.

[24] P. Painlevé, C. R. Acad. Sci. (Paris), 173, 677 (1921).

[25] A. Gullstrand, Arkiv. Mat. Astron. Fys., 16, 1 (1922).

[26] General Relativity, R. M. Wald, The University of Chicago Press (1984).

[27] Essential and inessential features of Hawking radiation, M. Visser, hep-th/0106111

[28] T. Jacobson and G. Kang, Class. Quantum Grav.10, L201 (1993).

[29] W. Wen, S. Men, and K. Lu, Phys. Rev. E 55, 3015 (1997).

[30] We could have considered $L=L\left(F, F^{*}\right)$ instead, where $F^{*} \equiv F_{\mu \nu}^{*} F^{\mu \nu}$. This case is studied in 6].

[31] The particular instance in which the vectors used as a basis in Eqn.25 are not linearly independent is discussed in 23]. 
[32] Note that a conformal factor to make $g_{11}=-1$ in Eq.(44) is needed. Consequently, the two metrics are actually conformally equivalent.

[33] Notice that we cannot take the limit $q \rightarrow 0$ in this expression or in any expression in which this one has been used.

[34] Note that this equation depends on $\chi^{(2)}$ through the expression for $r_{h}$, Eqn. [69]. 